\title{
North Carolina Medicaid Recipient Management Lock-In Program: The Pharmacist's Perspective
}

\author{
S. Rose Werth, BA; Nidhi Sachdeva, MPH, CHES; Andrew W. Roberts, PharmD; \\ Mariana Garrettson, MPH; Chris Ringwalt, PhD; Leslie A. Moss, MHA, CHES; \\ Theodore Pikoulas, PharmD, BCPP; and Asheley Cockrell Skinner, PhD
}

\begin{abstract}
BACKGROUND: The misuse and abuse of prescription opioids have become an urgent health issue in North Carolina (NC), particularly among Medicaid patients who suffer high rates of morbidity and mortality due to abuse and overdose. The NC Division of Medical Assistance (DMA) implemented a recipient management lock-in program, which limits identified patients for a 12-month period to 1 prescriber and 1 pharmacy for benzodiazepine, opiate, and certain anxiolytic prescriptions in order to prevent misuse and reduce overutilization of Medicaid benefits.
\end{abstract}

OBJECTIVES: To (a) evaluate pharmacists' perceptions of the implementation of the NC recipient management lock-in program (MLIP) and (b) determine how the beliefs and attitudes of pharmacists could promote or inhibit its success.

METHODS: We conducted 12 structured phone interviews with NC pharmacists serving lock-in patients. Interview responses were analyzed through construct analysis, which identified themes organized into 3 domains: organization and implementation, perceived effectiveness, and acceptability.

RESULTS: Most respondents reported a positive experience with the program but expressed doubt concerning its impact on prescription drug abuse. The program successfully utilized the pharmacist role as a gatekeeper of controlled substances, and the procedures of the program required no active effort on pharmacists' part. However, respondents suggested that the DMA improve communication and outreach to address pharmacists' lack of knowledge about the program's purpose and confusion over remediating problems that arise with lock-in patients. The DMA should also address the ways in which the program can interfere with access to health care and treatment, allow patients to see multiple physicians within the same clinic, and clarify procedures for patients whose complex health issues require multiple specialists.

CONCLUSIONS: Although possible improvements were identified, the NC MLIP has strong potential for success as it utilizes pharmacists' medication gate-keeping role, while minimizing the effort required for successful implementation.

J Manag Care Pharm. 2014;20(11):1122-28

Copyright $\odot 2014$, Academy of Managed Care Pharmacy. All rights reserved.

\section{What is already known about this subject}

Mortality among Medicaid recipients due to prescription drug abuse is 5 times that of the general population.

North Carolina Medicaid beneficiaries and providers had one of the highest rates of potentially fraudulent purchases of controlled substances in the the nation.
North Carolina implemented a narcotic lock-in program to address controlled substance misuse and reduce cost and utilization among Medicaid beneficiaries.

\section{What this study adds}

While many respondents reported an overall positive experience with the program, they doubted its impact on prescription drug misuse and abuse.

Educating pharmacists about the program's purpose and policies as well as addressing potential barriers to patient care may improve the program.

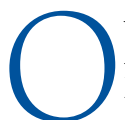
verdose and abuse of prescription drugs has become an increasingly urgent health issue in the United States. Most overdoses from prescribed medications result from nonmedical uses, which refers to both the misuse and abuse of prescription drugs. ${ }^{1}$ The national death rate due to unintentional poisoning increased by 91\% between 1999 and 2009 and by 213\% in North Carolina (NC) during the same period. ${ }^{2,3}$ Opioid analgesics, a class of controlled substances (CS) that includes methadone, codeine, hydrocodone, oxycodone, and morphine, among others, are the driving factor behind this increase. ${ }^{4}$ In the last decade, prescription painkillers have caused more deaths than either cocaine or heroin. ${ }^{5}$ The economic burden of prescription drug misuse and abuse is estimated to contribute $\$ 25$ billion to health care costs, $\$ 5$ billion to the justice system, and $\$ 25.6$ billion in workplace losses annually. ${ }^{6}$

Mortality among Medicaid recipients due to prescription drug abuse is 5 times that of the general population, and opioid nonmedical users are more likely to be covered by Medicaid than by any other insurance program. ${ }^{7.8}$ In 2009, the U.S. Government Accountability Office reported that NC Medicaid beneficiaries and providers had one of the highest rates of potentially fraudulent and abusive purchases of CS of any state in the nation. ${ }^{9}$

Efforts to prevent unintentional poisoning are reliant on the effective monitoring of prescription CS. The NC Division of Medical Assistance (DMA) implemented the NC Medicaid Recipient Management Lock-In Program (NC MLIP) to address issues of CS misuse and abuse in the NC Medicaid population. 
The goal of the program is to decrease the nonmedical use of opiates, benzodiazepines, and some anxiolytics by Medicaid recipients and to prevent recipient overutilization of Medicaid benefits. ${ }^{10}$ The program operates by identifying NC Medicaid recipients with 1 of the following criteria: (a) $>6$ prescription claims for an opioid medication in 2 consecutive months; (b) $>6$ prescription claims for a benzodiazepine or controlled anxiolytic medications in 2 consecutive months; or (c) $>3$ prescribers of these same CS in 2 consecutive months.

Providers, NC Medicaid administrators, and administrators at Community Care of North Carolina (CCNC), the state's managed care organization for Medicaid enrollees, can also nominate patients whom they think should be included in the program based on a subjective assessment of their risk behavior, although in practice, this option is rarely used. Once identified, Medicaid administrators lock patients into 1 prescriber and 1 pharmacy so that the recipient can only receive Medicaid-covered CS prescriptions from the providers to whom they are restricted. ${ }^{10}$ The patients will be locked in for a l-year time period after which they will be removed from the program if they no longer meet criteria..$^{10}$ There are 2,000 to 3,000 patients enrolled at any given time. The MLIP has already demonstrated effectiveness in an analysis conducted by the DMA in 2012, which showed that the program saved $\$ 5.2$ million over the first year and reduced the number of pain pills and anti-anxiety medications prescribed to MLIP patients by 2.3 million in only 3 months. ${ }^{11}$

The beliefs and attitudes of providers carrying out health interventions can either hinder or improve program effectiveness. ${ }^{12-16}$ Pharmacists, as the final intermediary between patient and prescription, are in a position to promote the successful operation of the program and identify opportunities for its improvement, provided they understand and support the process and implementation. The purpose of this study was to assess pharmacists' attitudes towards the MLIP and their experiences implementing the program by conducting in-depth structured interviews with NC pharmacists serving locked-in Medicaid patients.

\section{Methods}

\section{Respondents and Setting}

The sampling frame included all licensed pharmacists in NC. The NC Board of Pharmacy sent a solicitation e-mail to all licensed pharmacists, which included basic study information and a link to an initial screening survey. This survey was used to determine if respondents were practicing pharmacists with locked-in patients and to gauge their willingness to participate in phone interviews. From this screening, a list of 97 willing respondents was generated and prioritized according to the highest number of MLIP patients served. Two project staff members contacted and interviewed pharmacists from the prioritized list until the data were saturated to the point where no new information or themes emerged. In-depth phone interviews were conducted until thematic saturation was reached at a total of 12 pharmacists. Respondents practiced in a range of settings, including urban and rural pharmacies, independent and chain pharmacies, and clinic and hospital outpatient pharmacies.

\section{Structured Interviews}

Structured phone interviews were conducted using an Institutional Review Board (IRB)-approved topic guide (see Appendix, available in online article). Interview questions were intended to prompt pharmacists to report their experiences as the sole dispenser to a locked-in patient, as well as their experiences with the program, with particular regard to its impact on patient outcomes and access to health care. The interviews lasted 15 to 45 minutes, depending on the length of the respondent's answers and subsequent discussion. The interviews were audio-recorded, with each respondent's permission, to aid in subsequent data analysis.

\section{Data Management and Analysis}

Data were drawn from detailed notes that were taken by the interviewers; accuracy was confirmed using the audio recordings. Following thematic content analysis, ${ }^{17}$ each line or group of lines was coded with an identifier. Two members of the research team coded the first interview separately and created identifiers as they appeared within the data. The identifiers and codes were discussed and combined into 1 uniform codebook with the assistance of a third senior researcher. The remaining interviews were analyzed using the uniform codebook. If new codes arose from subsequent interviews, they were discussed and added to the codebook. The coded text was divided into themes as they emerged within the analysis, with quotes and sections from interviews selected to describe and exemplify each theme. The themes were used to examine pharmacists' perceptions of 3 domains: (1) program organization and implementation, (2) perceived effectiveness of the program, and (3) the program's acceptability to pharmacists and their patients.

This study was reviewed and approved by the University of North Carolina at Chapel Hill IRB.

\section{Results}

The screening survey yielded 97 respondents who met all selection criteria and were willing to participate in an interview. Interviews were arranged sequentially, prioritizing respondents who served the highest number of lock-in patients. Thematic saturation was reached with 12 in-depth structured interviews. References to CS indicate only those targeted by the NC MLIP: opiates, benzodiazepines, and certain anxiolytics.

Table 1 lists the 12 themes identified within the data, organizing each theme into 1 of the 3 domains. 
TABLE 1 Content Analysis, Domains, and Themes

\begin{tabular}{l|l|l|l}
\hline \multicolumn{2}{c}{ Domains } \\
\hline & \multicolumn{1}{c}{$\begin{array}{c}\text { Organization/ } \\
\text { Implementation }\end{array}$} & \multicolumn{1}{c}{$\begin{array}{c}\text { Perceived } \\
\text { Effectiveness }\end{array}$} & \multicolumn{1}{c}{ Acceptability } \\
\cline { 2 - 4 } & Notification & Overall opinion & $\begin{array}{l}\text { Difficulty of } \\
\text { implementation }\end{array}$ \\
\cline { 2 - 4 } & Selection process & Program impact & Impact on job roles \\
\cline { 2 - 4 } & Communication & $\begin{array}{l}\text { Impact on dispensing } \\
\text { patterns }\end{array}$ & $\begin{array}{l}\text { Unintended effects on } \\
\text { patients }\end{array}$ \\
\cline { 2 - 4 } & $\begin{array}{l}\text { Understanding of } \\
\text { program intent }\end{array}$ & Proposed changes & \\
\cline { 2 - 4 } & Problem remediation & & \\
\hline
\end{tabular}

\section{Domain 1: Organization and Implementation}

Notification. With the exception of 1 respondent, pharmacists received or were aware of letters sent from Medicaid informing them that their pharmacies had become the sole dispenser for a patient enrolled in the MLIP. Pharmacists can refuse a patient who has been locked-in, but none of the respondents had used this option. However, many respondents also depended on the claims process to alert them to locked-in patients. One pharmacist commented: "I have seen letters that come in the mail sometimes informing us of a patient that may or may not be locked in, but we generally rely on the [claims] transmission process of the Medicaid system."

Respondents reported very few problems with the notification process and transition into the program. A few pharmacists reported patients claiming to be unaware of their lock-in status, but the pharmacists were skeptical about these patients' reports that they had not been formally notified.

Selection Process. The majority of respondents stated that they did not believe any of their patients were mistakenly enrolled into the program. On the contrary, many believed that too few of their patients were locked in. One respondent stated: "I've seen a lot that have been mistakenly not enrolled in the program.... There's a lot of people falling through the cracks."

Only 3 other respondents described patients as unfit for the program. One pharmacist stated that up to $75 \%$ of his lockedin patients did not belong in the MLIP and went further, saying: "I don't know that we've ever had a scenario that I felt like the patient had been appropriately locked in with a prescriber."

Communication with Administrators and Managers. The main form of communication between pharmacists and program officials was Medicaid phone channels. If pharmacists needed to contact Medicaid administrators with questions about the program or problems with patients or claims, they had to call the Medicaid phone line and wait on hold to be redirected to an administrator in charge of the program or leave a message. Respondents expressed frustration about Medicaid's limited "bankers' hours." Many pharmacists reported waiting on hold for long periods of time or waiting for hours for a return phone call.
Communications between the DMA and each of the pharmacies did not flow through corresponding channels. Respondents described different protocols within pharmacies for handling messages and information from the state Medicaid program. One pharmacist reported that only 1 person within the pharmacy handled communications from Medicaid. A respondent who had previously served as a pharmacy manager mentioned that managers may not relay information about the program to their technicians. The level of support for the program from pharmacy management varied considerably.

Understanding of Program Intent. Respondents received mixed or incomplete messages about the goals and intentions of the MLIP. Ten of 12 respondents stated the explicit desired outcome that the program would ensure patients received their CS prescriptions from 1 prescriber and 1 pharmacist. Other perceived goals of the program included reduction of doctor and pharmacy shopping, minimization of Medicaid costs, prevention of prescription drug misuse and abuse, and prevention of adverse patient outcomes. However, 1 pharmacist expressed frustration that "They don't really tell us what the goal is. I don't know. I would guess cost, but it could be anything."

Half of respondents were confused about the boundaries between the MLIP and the NC Medicaid prescription capitation program. The capitation program required patients who filled 11 prescriptions of any drug category within a certain time period to opt-in to a locked-in single pharmacy if they needed another prescription that same month. The capitation program ended several months prior to our interviews. Respondents called it the "over 11" or "opt-in" program, reporting that many pharmacists use the terminology of the 2 programs interchangeably. Some respondents explained that their pharmacy computers used the same indicators for MLIP patients as were used for the previous program. A couple of respondents were unaware that the MLIP was an entirely separate program.

Problem Remediation. Respondents experienced difficulty when resolving problems they encountered with the program. A few pharmacists mentioned that drug shortages in the past year required patients to switch to a nonlock-in pharmacy that stocked their prescriptions. Respondents labeled this a "temporary unlock" or "secondary lock." Two pharmacists discussed their patients' need for exceptions in the case of holidays, closings, or travel and their wish that they could provide these patients with 3-day supplies in such situations. When encountering issues with prescribers, a number of pharmacists did not know of any readily available solutions, especially for incidents occurring on weekends. In 1 case, a respondent's patient was discharged from an emergency department (ED) and presented a CS prescription written by the ED doctor: "We can't [fill it]. There's no work around. What the patient is required to do is take the provider's prescription and contact that provider that's been approved to rewrite the order. To me, if it's a surgical patient, that could be something that causes them to come back to the hospital. Especially if it's on the weekend and the provider is not open 'til Monday. Really a lot of these hiccups come on the weekend." 


\section{Domain 2: Perceived Effectiveness}

Overall Opinion. Ten of 12 respondents reported positive experiences with the program but also volunteered flaws or areas for improvement. One pharmacist viewed the program as a temporary fix and said that many patients would "go back to their old lifestyle" after only a year in the program. Those who expressed the most positive opinions recognized the extent of the prescription drug epidemic and appreciated the program's efforts to mitigate it. Of the pharmacists interviewed, only 2 had explicitly negative evaluations of the program, with 1 calling it "one of the worst programs I've ever dealt with." These pharmacists believed that the MLIP was either ineffective or was actively harming their patients by restricting access to care. The discussion under the next section, "Domain 3: Acceptability" further addresses this effect.

Program Impact. With regard to specific program outcomes, 4 pharmacists believed that it reduced doctor shopping; 4 believed that it reduced misuse, abuse, and diversion of drugs; and 3 believed that it reduced Medicaid costs. Two other pharmacists stated that the program somewhat affected these outcomes but was not effective enough. Four respondents explicitly stated that it did not affect any abuse or misuse outcomes. A major issue brought up by 8 of the 12 respondents was that patients may circumvent the program by paying cash for their prescriptions. In a few instances, cash payments were used for prescriptions that respondents viewed as medically necessary even though they had been rejected by Medicaid. However, respondents primarily saw cash payments as a way for misusers and abusers to continue receiving excess prescriptions. One pharmacist stated: "I have a feeling if people are doctor hopping and shopping, they'll just learn to go to a store they've never been to and pay cash. I don't know how effective [the program] is at keeping controlled substances off the street that shouldn't be there."

Impact on Dispensing Patterns. Seven of the 12 pharmacists stated that the program had not altered how they monitored enrolled patients. The other 5 respondents treated lock-in patients differently, refusing to fill certain prescriptions, checking the state's Controlled Substance Reporting System before all fills, and verifying prescriptions with physicians. Four respondents believed that the program had increased their monitoring of all patients. One respondent particularly stressed this effect: "I think the idea that knowing that somebody else is watching and that there's a certain level of accountability changes it for everyone. If it's successful it should change your thought process across the board."

Proposed Changes. Respondents proposed a wide array of changes or improvements to the program. They most frequently requested improved communication with Medicaid. One respondent proposed changes to the Medicaid newsletter: "I would like to see the [lock-in policies] in the Medicaid newsletter come back out on a recurring basis at least a couple times a year just to remind pharmacy providers that there are options for patients."

One pharmacist proposed changes to address cash payments: "II would like it] if they had a line that would allow health care practitioners out there to submit things for potential abuse situations or diversion situations. When a man comes in locked into 1 store and his prescription is early and he offers to pay several hundred dollars in cash to get the prescription early — that should be reported somewhere."

Other recommended changes included expanding the program to enroll more patients; managing patients using chronic pain specialists; increasing pharmacist awareness and education about the program; locking patients into 1 clinic instead of 1 unique provider; and improving patients' transition out of the program.

\section{Domain 3: Acceptability}

Difficulty in Implemention. The MLIP program was not difficult for respondents to implement. Five of the 12 respondents specifically mentioned that their roles were passive or did not require effort outside of their normal duties.

Impact on Job Roles. Respondents honed in on 3 different roles they take on as pharmacists: as a caregiver, a gatekeeper, and as a business person. Pharmacists must maintain a balance between providing care to their patients and controlling the flow of prescription drugs within a community. With regard to controlling drug flow, 1 pharmacist described this tradeoff: "There's a wide spectrum. There's some who feel like they need to be the police. I don't think that's our role. I think we need to make the prescribers aware and be aware ourselves. You have to have some dialogue with the patients. If you just turn everyone away they'll go to multiple prescribers and pharmacies. They will learn how to work the system." Several pharmacists reported that the program had made their roles easier. One pharmacist expressed gratitude: "Really it makes my life a lot easier being the sole provider. I mean really it does because that takes the question out. So if they're only seeing 1 doctor and 1 drug store then there's that much more control." Aiding their role as caregivers, the program would alert respondents to when a patient needed counseling: "It really affirms in a busy pharmacy world, where you might be filling hundreds of prescriptions, that you need to stop, take a pregnant pause for a minute, and have a conversation."

Other pharmacists mentioned that acting as the sole dispenser allows them to improve adherence and track disease states. Many respondents saw the program as supportive of their business, insofar as the patients locked into their pharmacies increase their patient volume and sales. When asked about rejecting lock-in patients, 1 pharmacist replied: "I've never tried not to be locked in [i.e., involved in the lock-in program] because of course we're trying to increase prescription sales." For a few 
respondents, focusing on the program's potential to increase volume was an "economic reality" faced by independent pharmacies, which often struggle to make a profit against large chains.

Unintended Effects on Patients. Pharmacists were asked how the program affected their patients' access to care. Six respondents reported that the program could be a barrier to receiving pain treatment, mental health care, or general health care. While 6 respondents stated that the program had no effect on access, 3 of those 6 also described instances where patients had difficulty receiving medications or care at other points in the interview. Some of these problems arose from the type of physician a patient was locked in with: "I think what happened was a lot of patients were identified for the lock-in program because they had received short supplies of opiate pain relievers-either they were postsurgical, or they had an injury, an accident, or something. And, then they were locked in with this short-term-sometimes it was even an ED physician - and then they were locked out of their psychiatrists. And, then it would go the opposite way, too... people who had gotten locked in with their psychiatrists, but then their pain doctor was locked out and couldn't write their medicines."

Other access issues stemmed from the availability of physicians: "They make an appointment for their next visit and the prescriber they're locked in individually is not here. They see whoever is available to be seen next. They're not doctor shopping. They're just seeing whoever is there."

Availability of Medicaid also hindered access: "Any of those types of programs where stuff needs approval or assistance at the Medicaid level, that's a potential barrier in all of those cases. Not to say those programs aren't all good, but when you don't have 24-hour, 7-days-a-week coverage for those types of things, that is a barrier for some patients."

One particularly frustrated respondent summed up this issue as an inherent design flaw: "The program's not designed to keep the bad people away and the keep the good people getting what they need. It's just designed to keep the bad people away. It's hard to do both, but it can be done... The way it was done you ended up with a lot of people who probably would have benefited from being locked into 1 pain provider but were adversely affected on other parts of their treatment."

\section{Discussion}

The qualitative data ascertained through in-depth interviews with NC MLIP pharmacists revealed a wide range of experiences with, and opinions about, the lock-in program. While results may not be generalizable to all program pharmacists, its findings can provide insight into the program's strengths and weaknesses.

\section{Organization and Implementation}

While respondents valued the ease of enrollment, many believed that communication within the program was problematic. One pharmacist stated, "I think it would be a better program if all pharmacists knew exactly what we were trying to do." The program was initially announced to pharmacies through the DMA bulletin, while several subsequent bulletins issued reminders. ${ }^{10,18,19}$ CCNC clinical pharmacists have also done education and training with regards to the MLIP, especially with community pharmacies throughout the state. The work culture within some respondents' pharmacies may have interfered with their ability to receive these messages. If individual staff members handle Medicaid correspondence without communicating with their coworkers, or if pharmacy managers do not discuss the program's goals and features with their staff, widespread understanding of the program cannot be achieved.

The issues that respondents faced with problem remediation may be a result of their confusion about the program's goals and policies. Only 1 DMA bulletin mentioned how to handle lock-in exceptions. In an emergency situation, a 4-day supply of a CS may be provided to patients by a pharmacy or prescriber to which they are not locked in. ${ }^{10}$ The process for switching lock-in providers or handling other complications is not clearly available online nor was it described in any DMA bulletins. Only 1 pharmacist mentioned this policy; others merely expressed confusion. Respondents' dissatisfaction with Medicaid phone channels underscores program shortfalls in communications.

\section{Perceived Effectiveness}

The interviewed pharmacists gave mixed responses when asked whether the program had affected their CS dispensing practices. Some pharmacists reported increased monitoring of locked-in patients, but the majority stated that the program had not affected how they dispensed CS.

While interviews revealed both positive and negative aspects of the program, the recurring mention of cash payments for CS prescriptions outside of the program indicates a substantial weakness. While the scale of this problem is unknown, cash payments for CS prescriptions would undoubtedly counteract the programs' impact, outside of reducing Medicaid expenditures. Also, there has been some question as to whether all pharmacists are upholding the integrity of the program, with some possibly allowing patients to fill a CS with cash after receiving a rejected prescription claim from Medicaid. Fortunately, NC's prescription drug monitoring program, the Controlled Substances Reporting System, will soon be upgraded to include the method of payment used for all CS prescriptions in the system records. Awareness by pharmacists that a patient is enrolled in Medicaid should make it 
increasingly difficult for Medicaid patients to circumvent the MLIP by paying cash.

Several respondents stated that a 12-month lock-in does not allow sufficient time to establish long-term positive effects on recipient behavior. This time period does allow for CCNC clinical pharmacists and care managers to offer education to the patient, connect the patient with any needed substance abuse services, and provide more intensive care management services. ${ }^{20,21}$ The MLIP also lacks a transition period at the end of recipients' lock-in. Patients are re-enrolled if they still meet lock-in criteria. However, there is no transitory process or provision of resources for those who no longer qualify, even if their pharmacists believe they are still misusing or abusing CS.

\section{Program Acceptability}

Overall, program acceptability was high among study respondents, in large part because the automated Medicaid claims system minimized pharmacists' efforts in actively engaging the MLIP. The program also allowed pharmacists to track patient utilization behavior, prompted them to provide targeted patient counseling, and ensured continued patronage. However, the biggest concern to arise from these interviews was that the program may impede patients' access to care. For example, patients who are locked in with a single provider, as opposed to any provider within a given clinic, may be denied appropriate treatment if their prescribers are unavailable.

The program may be particularly harmful to patients with multiple diseases, such as chronic pain and psychiatric comorbidities, since these patients are more likely to see multiple specialists. Particular problems may arise from the program's definition of CS. The definition applies to opioid analgesics, which are used to treat acute and chronic pain, and to benzodiazepines and anxiolytics, which are most commonly used to treat anxiety disorders. While there is significant correlation between chronic pain and mental health issues, opioid analgesics are usually prescribed by different specialists than benzodiazepines or anxiolytics. ${ }^{22}$ While allowing patients to receive CS prescriptions from multiple providers may present a health risk, ${ }^{23}$ locking them into 1 provider may not be the most advantageous method to address complex health needs. For this reason, the DMA may be requested to allow for up to 2 providers for a single patient in situations in which 2 prescribers are being utilized (i.e., benzodiazepine prescribed by a psychiatrist and opioid medication prescribed by a pain specialist). It appears however, that pharmacists were not widely aware of this policy.

The MLIP has only 1 set of enrollment criteria for all opiate, benzodiazepines, and controlled anxiolytic users, treating them as a single, homogenous group. This strategy could lead to inappropriate patient enrollment, targeting patients with broadly specified criteria. Multiple studies have illustrated that prescription drug users comprise diverse subpopulations ${ }^{21-23}$ that include high school students; the elderly; street drug users; chronic pain, cancer, and acute pain patients; and patients receiving end-of-life care. ${ }^{24-26}$ The reason patients misuse CS also vary, ranging from poor patient education and undertreatment of pain to dependence and addiction, diversion, and desire for euphoria. ${ }^{24,26}$ The MLIP's enrollment criteria may not be effectively capturing these diverse subpopulations, although the ultimate goal for each group of patients would be the same: to reduce use and misuse of prescription medications, reduce unintentional overdoses, and promote the safe and effective use of these dangerous medications that can be addicting to all patients.

\section{Limitations}

The foundational strength of this study was the use of structured interviews, allowing the pharmacists surveyed to express a wide range of thoughts and opinions. The interview guide ensured that researchers covered key topics with pharmacists, while giving them the opportunity to freely express their opinions and concerns and raise new and unforeseen topics for discussion. The primary study limitation was the small sample size, leading to a potential limited generalizability of study results. Additionally, although we sent our request to all members of the NC Board of Pharmacy, the sample consisted primarily of outpatient community pharmacists.

\section{Conclusions}

This study revealed strengths and shortcomings of the NC MLIP. The program holds promise for success as it utilizes pharmacists' medication gate-keeping role, while minimizing the effort required from pharmacists for successful implementation. The program can also improve pharmacists' awareness of prescription drug misuse and abuse among their patients and improve their ability to prevent and combat it. However, more outreach is needed by the DMA in order to educate pharmacists about the MLIP's policies and underlying purpose. Improved communication between pharmacies and the DMA is also necessary for program success. This is particularly true when issues with locked-in patients arise (e.g., on weekends). Resolving these issues may require new or improved methods of communication. Examples of methods that may be beneficial include extended access to Medicaid phone channels; a direct phone line for problems pharmacies face with locked-in patients; or set protocols for them to follow when common issues arise. The flexibility of the program should also be enhanced, allowing patients to see multiple prescribers within the same clinic. While the NC MLIP addresses an urgent health issue within the NC Medicaid population, further refinements have the potential to substantially enhance its impact. 


\section{Authors}

S. ROSE WERTH, BA, is Program Specialist, Partnership for a DrugFree NC, Durham, North Carolina. NIDHI SACHDEVA, MPH, CHES, is Project Manager; MARIANA GARRETTSON, MPH, is Research Scientist; and CHRIS RINGWALT, PhD, is Senior Scientist, Injury Prevention Research Center, University of North Carolina at Chapel Hill. ANDREW W. ROBERTS, PharmD, is PhD Candidate, Division of Pharmaceutical Outcomes and Policy, UNC Eshelman School of Pharmacy, and Postdoctoral Fellow, Cecil G. Sheps Center for Health Services Research, University of North Carolina at Chapel Hill; LESLIE A. MOSS, MHA, CHES, is Data Coordinator, Division of Medical Assistance, North Carolina Department of Health and Human Services, Raleigh; THEODORE PIKOULAS, PharmD, BCPP, is Associate Director of Behavioral Health Pharmacy Programs, Community Care of North Carolina, Raleigh; and ASHELEY COCKRELL SKINNER, PhD, is Associate Professor of Pediatrics, University of North Carolina at Chapel Hill.

AUTHOR CORRESPONDENCE: Asheley Cockrell Skinner, PhD, University of North Carolina at Chapel Hill School of Medicine, 231 MacNider, 229B, CB 7225, Chapel Hill, NC 27599.

Tel.: 919.843.9941; E-mail: asheley@unc.edu.

\section{DISCLOSURES}

This research was supported by Cooperative Agreement CDC U01 CE00216001, Building Interdisciplinary Research Careers in Women's Health (BIRWCH) Training Grant NIH K12 HD00144l (Skinner), CTSA Grant UL1TR000083, the UNC Injury Prevention Research Center, and a National Research Service Award Post-Doctoral Traineeship from the Agency for Health Care Research and Quality sponsored by the Cecil G. Sheps Center for Health Services Research, 5 T32 HS000032 (Roberts). The authors have no conflicts of interest to disclose.

Study concept and design was primarily contributed by Garrettson, Ringwalt, and Skinner, with assistance from Sachdeva and Werth. Data collection was primarily the responsibility of Werth and Sachdeva, with assistance from Garrettson and Ringwalt, and Pikoulas and Werth interpreted the data, with assistance from the rest of the authors. The manuscript was written by Werth, Roberts, and Ringwalt, assisted by the rest of the authors, and revision was carried out by Moss and Skinner, assisted by the rest of the authors.

\section{ACKNOWLEDGMENTS}

The authors would like to thank the North Carolina Board of Pharmacy for its assistance in recruitment.

\section{REFERENCES}

1. Substance Abuse and Mental Health Services Administration, Center for Behavioral Health Statistics and Quality. Highlights of the 2010 Drug Abuse Warning Network (DAWN) findings on drug-related emergency department visits. The DAWN Report. July 2, 2012. Rockville, MD. Available at: http://www.samhsa.gov/data/2k12/DAWN096/SR096EDHighlights2010.pdf. Accessed August 5, 2014.

2. Centers for Disease Control and Prevention. Prevent unintentional poisoning. 2012. Available at: http://www.cdc.gov/Features/PoisonPrevention/. Accessed August 5, 2014.

3. Ford MD. Unintentional poisoning in North Carolina: an emerging public health problem. N C Med J. 2010;71(6):542-46.

4. Warner M, Chen LH, Makuc DM. Increase in fatal poisonings involving opioid analgesics in the United States, 1999-2006. NCHS data brief, no 22. Hyattsville, MD: National Center for Health Statistics; 2009. Available at: http://www.stoprxdrugabuse.org/2009_9_30_CDC-_Opioid_ Analgesics_1999-2006_US.pdf. Accessed August 5, 2014.

5. Paulozzi LJ, Budnitz DS, Xi Y. Increasing deaths from opioid analgesics in the United States. Pharmacoepidemiol Drug Saf. 2006;15(9):618-27.
6. Birnbaum HG, White AG, Schiller M, Waldman T, Cleveland JM, Roland CL. Societal costs of prescription opioid abuse, dependence, and misuse in the United States. Pain Med. 2011;12(4):657-67.

7. Centers for Disease Control and Prevention. Overdose deaths involving prescription opioids among Medicaid enrollees-Washington, 2004-2007. MMWR Morb Mortal Wkly Rep. 2009;58(42):1171-75. Available at: http://www.cdc.gov/ mmwr/preview/mmwrhtml/mm5842al.htm. Accessed August 5, 2014

8. Centers for Disease Control and Prevention. Issue brief: unintentional drug poisoning in the United States. MMWR Morb Mortal Wkly Rep. 2010;59(10):300. Available at: http://www.cdc.gov/homeandrecreationalsafety/pdf/poison-issue-brief.pdf. Accessed August 5, 2014.

9. U.S. Government Accountability Office. Medicaid: fraud and abuse related to controlled substances identified in selected states. GAO report number GAO-09-957. 2009. Available at: http://www.gao.gov/assets/300/294715. html. Accessed August 5, 2014.

10. North Carolina Division of Medical Assistance. Implementation of a recipient management lock-in program. August 2010 Medicaid Bulletin. Available at: http://www.ncdhhs.gov/dma/bulletin/0810bulletin.htm\#lock. Accessed August 5, 2014.

11. North Carolina Department of Health and Human Services. 2.3 Million pills off the streets, \$5.2 million saved by narcotics lock-in. May 14, 2012. Available at: http://www.ncdhhs.gov/pressrel/2012/2012-05-14_pills_off_ streets.htm. Accessed August 5, 2014.

12. Aarons GA. Measuring provider attitudes toward evidence-based practice: consideration of organizational context and individual differences. Child Adolesc Psychiatr Clin N Am. 2005;14(2):255-71, viii.

13. Aarons GA, Palinkas LA. Implementation of evidence-based practice in child welfare: service provider perspectives. Adm Policy Ment Health. 2007;34(4):411-19.

14. Cornuz J, Ghali WA, Carlantonio D, Pecoud A, Paccaud F. Physicians' attitudes towards prevention: importance of intervention-specific barriers and physicians' health habits. Fam Pract. 2000;17(6):535-40.

15. Glisson C, Landsverk J, Schoenwald S, et al. Assessing the organizational social context (OSC) of mental health services: implications for research and practice. Adm Policy Ment Health. 2008;35(1-2):98-113.

16. Kushner RF. Barriers to providing nutrition counseling by physicians: a survey of primary care practitioners. Prev Med. 1995;24(6):546-52.

17. Attride-Stirling J. Thematic networks: an analytic tool for qualitative research. Qual Res. 2001;1(3):385-405.

18. North Carolina Division of Medical Assistance. Recipient management lock-in program emergency fill. October 2010 Medicaid Bulletin. Available at: http://www. ncdhhs.gov/dma/bulletin/1010bulletin.htm\#lock. Accessed August 5, 2014.

19. North Carolina Division of Medical Assistance. Recipient opt-in program and monthly prescription limits. February 2013 Medicaid Bulletin. Available at: http:// www.ncdhhs.gov/dma/bulletin/0213bulletin.htm\#optin. Accessed August 5, 2014 20. Jackson CT, Trygstad TK, DeWalt DA, DuBard CA. Transitional care cut hospital readmissions for North Carolina Medicaid patients with complex chronic conditions. Health Aff (Millwood). 2013;32(8):1407-15.

21. Chisholm-Burns MA, Kim Lee J, Spivey CA, et al. US pharmacists' effect as team members on patient care: systematic review and meta-analyses. Med Care. 2010;48(10):923-33.

22. Gureje O. Psychiatric aspects of pain. Curr Opin Psychiatry. 2007;20(1):42-46. 23. Centers for Disease Control and Prevention. CDC grand rounds: prescription drug overdoses-a U.S. epidemic. MMWR Morb Mortal Wkly Rep. 2012;61(1):10-13 Available at: http://www.cdc.gov/mmwr/preview/mmwrhtml/mm610la3.htm. Accessed August 5, 2014.

24. Green TC, Black R, Grimes Serrano JM, Budman SH, Butler SF. Typologies of prescription opioid use in a large sample of adults assessed for substance abuse treatment. PLoS One. 2011;6(11):e27244.

25. Ghandour LA, Martins SS, Chilcoat H. Understanding the patterns and distribution of opioid analgesic dependence symptoms using a latent empirical approach. Int J Methods Psychiatr Res. 2008;17(2):89-103.

26. Davis WR, Johnson BD. Prescription opioid use, misuse, and diversion among street drug users in New York City. Drug Alcohol Depend. 2008;92(1-3):267-76. 


\section{APPENDIX Interview Guide}

\section{PROVIDER INTERVIEW GUIDE}

Hello, my name is I am with the Injury Prevention Research Center at UNC Chapel Hill. I am calling to follow up with the interview about the Medicaid Lock-In Program that we scheduled for today. Is now still a good time for a 20- to 30-minute interview? (If not, reschedule.)

Great. Did you receive the fact sheet in the confirmation e-mail? I would like to review that briefly with you before we start. (Go over fact sheet.)

What questions, if any, do you have about the study or the fact sheet?

Please keep in mind that this interview is completely voluntary; there are no right or wrong answers-so please answer as honestly and best as you can; your name will not be linked to your responses; and you have the option to stop the interview at any time.

CONFIRM CONSENT: Are you still interested in participating? Would it be all right to begin the interview now?

\section{QUESTIONS}

My first questions are about the processes involved in becoming a NC MLIP patient's sole provider:

1. How do you get notified that you have been selected as a sole provider for an enrolled patient?

2. Can you decide whether to accept or not? If so,

a. How do you decide whether to accept a patient or not?

b. How do you communicate with Medicaid about your decision?

c. If you have declined to accept a patient, how did the Medicaid administrator respond?

3. Do you believe that any of the patients you serve have been mistakenly, or inappropriately, enrolled?

a. If so, what was the nature of the mistake?

4. What challenges, if any, have you had with any aspect of this process?

Now, I have several questions about your experience of treating patients who are enrolled in the NC MLIP:

5. We understand from the online survey that you have been the provider for XX patients in the MLIP, and you identified your overall experience with the program as XX on a scale of 1 to 6 . Is this still accurate?

a. (For people who have more than 1 patient locked in) What were the most common patient characteristics or circumstances that led to their initial enrollment in the program?

6. Please describe your characterization of your experience as positive and/or negative. Could you tell me more about what makes you characterize your experience that way?

7. If you were going to give advice to another physician/pharmacist who is accepting the role of sole provider, what would you say?

8. If you could make 1 change to the program that would make it easier for providers like you, what would that be?

My last questions are about your perceptions of the impact of this program on your patients and on you:

9. How does this program affect a patient's access to pain care? (Probe: Does the program improve or impair patients' effective pain management?)

10. How does this program affect a patient's access to mental health care? (Probe: Does the program improve or impair patients' mental illness management?)

11. How does this program affect a patient's access to all other medical care, if at all?

12. How does this program affect a patient's health?

13. How has this program changed the way that you prescribe/dispense controlled substances to patients who are enrolled in the program?

14. How has this program changed the way that you prescribe/dispense controlled substances to other patients?

15. What else about the implementation or impact of this program would you like to share? 